\title{
DISTÚRBIOS OSTEOMUSCULARES RELACIONADOS AO TRABALHO EM ENFERMEIROS DE UM HOSPITAL PÚBLICO EM MANAUS-AM
}

Work-related musculoskeletal disorders in nurses at a public hospital in Manaus-AM Desafios

Trastornos musculoesqueléticos relacionados con el trabajo en enfermeras de un hospital público de Manaus-AM

João Viriato Mazalo ${ }^{* 1}$, Bruno Mori ${ }^{2}$, Thays Ribeiro De Paulo ${ }^{3}$, Querolin Natacha Pinheiro $^{3}$, Antônio Luiz Boechat ${ }^{1,2}$

${ }^{1}$ Programa de Pós-Graduação em Ciências da Saúde - Universidade Federal do Amazonas, Manaus- AM, Brasil.

${ }^{2}$ Programa de Pós-Graduação em Imunologia Básica e Aplicada - Universidade Federal do Amazonas, ManausAM, Brasil.

${ }^{3}$ Graduada em Enfermagem pela Faculdade Estácio do Amazonas. Manaus- AM, Brasil.

*Correspondência: Faculdade de Medicina, Universidade Federal do Amazonas, Rua Afonso Pena, 1053 - Centro, Manaus - AM, CEP: 69020-160. E-mail: joaomazalo@gmail.com.

\section{RESUMO}

Os distúrbios osteomusculares relacionado ao trabalho são caracterizados pelo comprometimento e desgaste das estruturas do sistema musculoesquelético, em consequência das condições precárias de trabalho, má postura, carga horária de trabalho excessiva e movimentos repetitivos. Esta pesquisa teve como objetivo conhecer os distúrbios osteomusculares relacionados ao trabalho em enfermeiros. Trata-se de um estudo transversal com abordagem quantitativa. A amostra foi composta por 25 enfermeiros do Hospital e Pronto Socorro 28 de Agosto, Manaus-AM. Os dados foram coletados através de um questionário aplicado aos enfermeiros, contendo variáveis sócio demográficas, ocupacionais e o Questionário Nórdico de Sintomas Osteomusculares (QNSO), e posteriormente realizou-se análise descritiva. A prevalência dos sintomas osteomusculares foi de $56 \%$, sendo que $85,7 \%$ relataram que os sintomas pioravam com o exercício da enfermagem, sendo estes, casos sugestivos de distúrbios osteomusculares relacionados ao trabalho. As regiões anatômicas mais afetadas pelos sintomas foram a lombar $72 \%$, seguida pelos ombros e pés com $68 \%$, respectivamente. Concluiu-se que os enfermeiros entrevistados apresentavam casos sugestivos de distúrbios osteomusculares relacionados ao trabalho, pois a sintomatologia piorava com a prática da enfermagem. Deste modo, para evitar o afastamento temporário ou até mesmo definitivo destes profissionais por complicações de saúde, é necessário criar condições de trabalho adequadas que possibilitem prevenir o aparecimento dos distúrbios osteomusculares.

Palavras-chave: Doenças musculoesqueléticas, Equipe de enfermagem, Saúde do trabalhador.

\section{ABSTRACT}

Work-related musculoskeletal disorders are characterized by impairment and wear of the structures of the musculoskeletal system, as a result of poor working conditions, poor posture, excessive workload and repetitive movements. This research aimed to find out about musculoskeletal disorders related to work in nurses. This is a cross-sectional study with a quantitative approach. The sample consisted of 25 nurses from the Hospital e Pronto Socorro 28 de Agosto, Manaus-AM. Data were collected through a questionnaire applied to nurses, containing socio-demographic and occupational variables and the Nordic Musculoskeletal Questionnaire (QNSO), and subsequently, a descriptive analysis was performed. The prevalence of musculoskeletal symptoms was 56\%, with 
$85.7 \%$ reporting that the symptoms worsened with nursing practice, which are suggestive of work-related musculoskeletal disorders. The anatomical regions most affected by the symptoms were the lumbar 72\%, followed by the shoulders and feet with $68 \%$, respectively. It was concluded that the nurses interviewed presented cases suggestive of musculoskeletal disorders related to work, as the symptoms worsened with the practice of nursing. Thus, to avoid the temporary or even permanent removal of these professionals due to health complications, it is necessary to create adequate working conditions that make it possible to prevent the onset of musculoskeletal disorders.

Keywords: Musculoskeletal diseases, Nursing team, Occupational health.

\section{RESUMEN}

Los trastornos musculoesqueléticos relacionados con el trabajo se caracterizan por el deterioro y desgaste de las estructuras del sistema musculoesquelético, como resultado de malas condiciones de trabajo, mala postura, carga de trabajo excesiva y movimientos repetitivos. Esta investigación tuvo como objetivo conocer los trastornos musculoesqueléticos relacionados con el trabajo en enfermeras. Se trata de un estudio transversal con enfoque cuantitativo. La muestra estuvo conformada por 25 enfermeras del Hospital e Pronto Socorro 28 de Agosto, Manaus-AM. Los datos se recogieron mediante un cuestionario aplicado a enfermeras, que contenía variables sociodemográficas y ocupacionales y el Cuestionario Nórdico Musculoesquelético (QNSO), y posteriormente se realizó un análisis descriptivo. La prevalencia de síntomas musculoesqueléticos fue del 56\%, y el 85,7\% informó que los síntomas empeoraron con la práctica de enfermería, lo que sugiere trastornos musculoesqueléticos relacionados con el trabajo. Las regiones anatómicas más afectadas por los síntomas fueron las lumbares $72 \%$, seguidas de hombros y pies con $68 \%$, respectivamente. Se concluyó que los enfermeros entrevistados presentaron casos sugestivos de trastornos musculoesqueléticos relacionados con el trabajo, ya que los síntomas empeoraron con la práctica de la enfermería. Así, para evitar la baja temporal o incluso permanente de estos profesionales por complicaciones de salud, es necesario crear unas condiciones laborales adecuadas que permitan prevenir la aparición de trastornos musculoesqueléticos.

Descriptores: Enfermedades musculoesqueléticas, Equipo de enfermería, Salud laboral.

\section{INTRODUÇÃO}

Desde os anos passados até aos dias atuais muitos trabalhadores quando submetidos a intensas e longas jornadas de trabalho apresentam com o tempo diversos e distintos sintomas que implicam em ações trabalhistas (LUCCA, 2017). A cada dia que passa há necessidade de aprimorar conhecimentos que podem apoiar a cada classe trabalhadora a ultrapassar as dificuldades que advém de tais distúrbios. Assim, a esse conjunto de sintomas é dado a sigla LER- Lesões por esforços repetitivos, e posteriormente, DORTDistúrbio Osteomuscular Relacionada ao Trabalho (FERRI; XAVIER, 2015).

O distúrbio osteomuscular relacionado ao trabalho (DORT) é conceituado por muitos estudiosos como um problema de saúde pública que afeta os enfermeiros (PAULA; AMARAL, 2019). DORT é uma designação de um grupo de doenças osteomusculares, que têm repercussões sociais, bem como psicológicas (ALMEIDA et al., 2016; MARTINS; CORREA, 2017). É caracterizado pelo desgaste das estruturas do sistema musculoesquelético, sendo causadas por consequência de: condições precárias de trabalho, repetitividade, má postura, resistência pessoal, esforço, velocidade no cumprimento da atividade, estado psicossocial do indivíduo, bem como outros fatores externos e internos que podem influenciar tais como: iluminação, ruídos, frio, vibração, umidade, espaço físico (COSTA; DIAS, 2015; SANTOS; ANDRADE, 2011). O DORT sobrecarrega os nervos, articulações, cartilagens, discos intervertebrais, tendões e músculos, sendo que muitas das vezes prejudica principalmente os membros superiores e o pescoço (PAULA; AMARAL, 2019; ROCHA et al., 2017). Esta sobrecarga pode desencadear: tendinite, 
tenossinovite, dedo em gatilho, compressão nos nervos, bursite, epicondilite, síndrome do túnel do carpo, cistos sinoviais (MELO; ASSIS, 2018; SANTOS; ANDRADE, 2011). Por isso, alguns sintomas são comuns: queixas de incômodos ou dores lombares, ombros, joelhos e região cervical, perda de força muscular, fisgadas, peso e cansaço no braço, formigamento nos pés e mãos, dormência, dor, choques, inchaço, edemas, pele avermelhada, sensação de peso, calor localizado, ansiedade, irritabilidade, distúrbios de sono e fadiga crônica (BRITO; VENENO, 2015; FELLI et al., 2015; SANTOS; ANDRADE, 2011).

Para o diagnóstico DORT realiza-se a análise clínica em consonância com exames de imagem como eletroneuromiografia, tomografia computadorizada, ressonância magnética, ultrassonografia. Podendo ser tratada após o diagnóstico preciso, pois DORT é composto por um conjunto de doenças que se não for bem diagnosticada o tratamento não será eficaz (REIS, 2016). Sua terapêutica abrange não só procedimentos cirúrgicos, mas também infiltrações, órteses, fisioterapia, medicamentos (anti-inflamatórios e analgésicos) (PINHATTI et al., 2018).

Existe um elevado nível de preocupação em relação ao DORT, porque ela pode acarretar a incapacidade do trabalhador realizar suas atividades, causando o afastamento temporário ou até mesmo definitivo, consequentemente gerando um custo alto com tratamentos, substituições ou até mesmo indenizações (SOUSA et al., 2015).

Os profissionais de enfermagem desenvolvem suas atividades em diversos locais, dentre os quais as instituições hospitalares, onde realizam atividades de forma contínua, que exigem atenção constante, esforço físico, posições inadequadas, movimentos repetitivos, excessiva carga horária de trabalho, tornando-os expostos a diversos fatores de riscos ocupacionais e a um nível elevado de predisposição ao risco de adoecimento no trabalho (LELIS et al., 2012).

Dentro do grupo de profissionais de saúde empregados em hospitais, a enfermagem é mais propensa a DORT, devido a posições corporais desajeitadas por longos períodos, que podem causarlhes danos futuros, pois muitas vezes descuidam-se da própria saúde por estarem preocupados em satisfazer as funções instituídas para o cargo que ocupam (CARGNIN et al., 2019; DUARTE et al., 2012)

O principal fator de risco a etiologia das DORT em enfermeiros, é o manuseio dos pacientes, devido às posições extremas adotadas durante a prestação destes cuidados. Após várias pesquisas foi comprovado que é necessário dar atenção não só as atividades ligadas a manejo e transferência de pacientes, mas também as demais atividades de trabalho que requerem movimentos repetitivos e 58 precisos com altas exigências de atenção nos procedimentos realizados pelos enfermeiros no ambiente de trabalho (FONSECA et al., 2006; SOUSA et al., 2015). Os enfermeiros apresentam uma alta prevalência de DORT na região lombar, seguida dos membros superiores e dor nos joelhos (SERRANHEIRA et al., 2012).

Diante dos argumentos expostos acima, tornase relevante conhecer os distúrbios osteomusculares relacionados ao trabalho em enfermeiros do Hospital e Pronto Socorro 28 de Agosto, cidade de Manaus, estado do Amazonas.

\section{MATERIAIS E MÉTODOS}

Realizou-se um estudo transversal, com abordagem quantitativa, no Hospital e pronto Socorro 28 de Agosto, na cidade de Manaus, estado do Amazonas. Utilizou-se a amostragem por 
conveniência e adotou-se como critérios de inclusão, todos os enfermeiros que trabalhavam no Hospital por um período mínimo de seis (06) meses, que aceitaram assinar o termo de consentimento; sendo excluídos enfermeiros com critérios elegíveis para o estudo, mas que por algum motivo mostraram-se indisponíveis em participar no estudo. Durante o mês de setembro e outubro, 25 enfermeiros aceitaram participar do estudo e responderam as questões de forma completa.

Os dados foram obtidos, após a assinatura do consentimento, através de um questionário, composto por perguntas com variáveis sócio demográficas e ocupacionais com questões como, presença ou não de duplo vínculo empregatício, quantidade de carga horária de trabalho, presença ou ausência de dores osteomusculares, percepção sobre a piora ou não da dor com o exercício da atividade de enfermagem, e para sintomas pelo Questionário Nórdico de Sintomas Osteomusculares (QNSO) validado para a população brasileira (AMARAL et., 2002), um instrumento que contém múltiplas escolhas quanto à ocorrência de sintomas nas diversas regiões anatômicas.

Os dados foram organizados em planilha no software: Microsoft Office Excel para gerenciamento dos dados. Foi realizada uma análise descritiva para avaliar o perfil da amostra segundo as variáveis de estudo, sendo que para as variáveis categorias, utilizou-se tabela de frequência absoluta (n) e relativa $(\%)$.

O estudo respeitou a Resolução $\mathrm{n}^{\circ}$ 466, de 12 de dezembro de 2012 do Conselho Nacional de Saúde, durante todo percurso da pesquisa, atentado aos aspectos éticos e legais vigentes. A pesquisa foi aprovada pelo Comitê de Ética e Pesquisa da Faculdade Estácio do Amazonas sob o $\mathrm{n}^{\circ}$ 028336/2016 conforme Certificado de Apresentação para Apreciação Ética n ${ }^{0}$ 61404816.2.0000.5037.

\section{RESULTADOS E DISCUSSÃO}

Em relação às características sócio demográficas houve maior predominância do sexo feminino $(80,7 \%)$, sendo grande parte com a faixa etária de 31 a 45 anos (60\%). Quanto à composição familiar, $52 \%$ eram casados ou viviam em união estável e 76\% possuíam pelo menos um filho.

Nota-se que as caracterizações dos dados relacionados ao sexo coincidem com o perfil dos enfermeiros no Brasil relatados pela literatura, no que concerne a população predominante feminina. A enfermagem é conhecida não somente pela sua formação em ser na sua maioria do sexo feminino, mais também pelas suas atribuições específicas (FIOCRUZ/COFEN, 2013).

As atribuições culturais dadas a cada sexo sempre influenciaram nas escolhas profissionais, e concepções machistas como "o homem é superior a mulher", fizeram com que a enfermagem fosse vista como fácil, frágil e subordinada, razão pela qual, muitos homens optam por não ingressar em profissões tidas como femininas, salientando e reforçando essa divisão sexista nas profissões e sua hierarquização, onde o homem está no topo, e consequentemente deve exercer profissões de maior predominância e notoriedade. Assim, os homens que não seguem essa tendência, são discriminados por serem considerados inferiores (CUNHA; SOUSA, 2017).

$\mathrm{O}$ ambiente de trabalho em enfermagem deve considerar as diferenças fisiológicas entre ambos os sexos para evitar os DORT. Assim, os trabalhos que necessitem de aplicação da força, como o manuseio e transporte de pacientes em hospitais, deve respeitar as diferenças fisiológicas entre ambos os sexos, distribuindo $\mathrm{o}$ atendimento dos pacientes aos enfermeiros levando em consideração o peso corporal, separando os pacientes mais leves (para profissional 
de enfermagem do sexo feminino) dos mais pesados (para profissional de enfermagem do sexo masculino), criando condições para que todos deem o seu melhor contributo, sem comprometer a sua integridade física e respeitando as suas limitações (REIS, 2016).

A equipe de enfermagem é composta por jovens, adultos e indivíduos mais velhos. O processo de envelhecimento tem implicações no trabalho de enfermagem com a diminuição da força, flexibilidade, tempos de reação e da massa óssea, o que consequentemente pode resultar em maior risco de lesões ou mesmo retirada destes profissionais. A adaptação do ambiente de trabalho em alguns casos é necessária para criar condições que favorecem a continuidade dos enfermeiros mais experientes no setor, pois quando o trabalho se torna mais difícil por questões inerentes a limitação física, auditiva e da visão, são mais propensos a desistir da profissão (MATT et al., 2015).

Tabela 1. Distribuição dos Enfermeiros segundo características de carga horária de trabalho e duplo vínculo empregatício

\begin{tabular}{lcc}
\hline Variável & FA (n) & FR(\%) \\
\hline $\begin{array}{l}\text { Duplo vínculo } \\
\text { empregatício }\end{array}$ & & \\
Não & 12 & 48 \\
Sim & 13 & 52 \\
\hline Carga horária de & & \\
trabalho & & \\
$<40$ horas & 9 & 36 \\
$40-60$ horas & 14 & 56 \\
$>60$ horas & 2 & 8 \\
\hline
\end{tabular}

Na Tabela 1, observa-se que dos 25 enfermeiros entrevistados, $52 \%$ afirmaram possuir duplo vínculo empregatício. No que concerne a carga horária semanal, $56 \%$ dos enfermeiros relataram ter uma carga horária de 40-60 horas.

É importante compreender que os enfermeiros, técnicos e auxiliares de enfermagem no Brasil fazem parte de uma profissão caracterizada por ter sobrecarga horária de trabalho, frequentemente justificada por falta de pessoal, o que culmina com um ritmo exaustivo de trabalho, em regime de plantões, carga horária elevada, trabalho noturno, e faz com que os profissionais busquem mais de um emprego ou sejam estimulados pelo pagamento de horas extras, visto que os salários são baixos no setor de enfermagem (BEZERRA; COSTA, 2016; FRANÇA et al., 2012)

A exposição dos trabalhadores a carga horária de trabalho excessiva semanal gera processo de desgastes e suas consequências é uma medida que transcende à promoção e proteção da saúde dos trabalhadores de enfermagem, uma vez que gera problemas de saúde, onde aparecem com maior frequência os distúrbios osteomusculares, as doenças infecciosas e parasitárias e os transtornos mentais e comportamentais (FELLI et al., 2015).

Os turnos rotativos de trabalho contrários ao ritmo biológico do organismo, associado às longas jornadas, à baixa remuneração, ao frequente emprego duplo, ao desenvolvimento de tarefas que demandam sobrecarga horária laboral, podem deixar os enfermeiros suscetíveis a contrair o DORT (FRANÇA et al., 2012; LUCCA; RODRIGUES, 2015).

Analisando a Tabela 2, com base nos resultados dos Questionário Nórdico de Sintomas Osteomusculares constatou-se que, 56\% (14) dos enfermeiros afirmaram sentir algum tipo de dor osteomuscular, dos quais $85,7 \%$ (12) relataram que as dores estavam relacionadas com o trabalho, isto é, 
pioravam com o exercício da atividade de enfermagem, sendo estes, casos sugestivos de distúrbios osteomusculares relacionados ao trabalho. O espaço profissional da enfermagem é um campo que cotidianamente lida com distintas atividades, que em muitas vezes, coloca os enfermeiros em situações de desafios constantes.

Tabela 2. Respostas dos enfermeiros sobre a presença ou ausência de dores osteomusculares e relação da percepção sobre o agravamento ou não das dores durante o exercício da enfermagem

\begin{tabular}{l|c|c}
\multicolumn{1}{c|}{ Variável } & FA(n) & FR(\%) \\
\hline $\begin{array}{l}\text { Presença de dores } \\
\text { osteomusculares }\end{array}$ & \\
Sim & 14 & 56 \\
Não & 11 & 44 \\
Total & 25 & 100 \\
\hline Piora das dores no exercício da & & \\
enfermagem & & \\
Sim & 12 & 85,7 \\
Não & 2 & 14,3 \\
Total & 14 & 100 \\
\hline
\end{tabular}

Os enfermeiros acabam adoecendo e ficando cada vez mais afastados da sua profissão por fatores que influenciam o estado de saúde, com destaque para doenças do sistema osteomuscular, e do tecido conjuntivo, bem como por transtornos mentais e comportamentais (BAPTISTA et al., 2018).

$\mathrm{O}$ adoecimento provocado pelos distúrbios osteomusculares pode acarretar alterações na realização de atividades cotidianas, constituindo causa comum de afastamento do trabalho e com consequências financeiras significativas em razão da compensação de trabalhadores e despesas médicas, além de prejuízo a sociedade e qualidade de vida destes profissionais (MASCARENHAS; NOVAES, 2015).

Gráfico 1. Distribuição dos sintomas referentes as dores osteomusculares dos enfermeiros nas regiões anatômicas, com base no QNSO ( $\mathrm{n}=14)$.

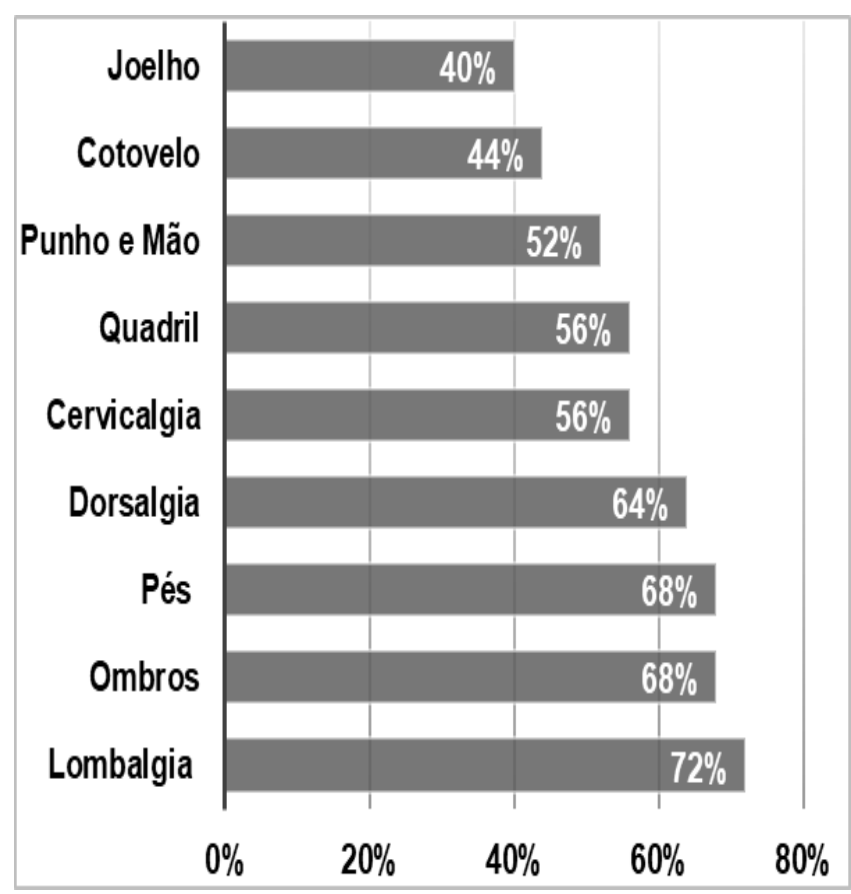

Com base no Questionário Nórdico de Sintomas Osteomusculares (QNSO), as regiões anatômicas mais afetadas pelos sintomas osteomusculares foram a lombar $72 \%$, seguida pelos ombros e pés com $68 \%$, respectivamente, como ilustra o Gráfico 1. A magnitude de DORT encontrada nesta pesquisa, pode ter sido influenciada pela rotina laboral e/ou condições de trabalho na qual os enfermeiros foram expostos neste hospital, aliadas ao desejo de querer melhorar as condições de salário com duplo vínculo empregatício, que incrementa diretamente a sobrecarga laboral, e consequentemente carga horária de trabalho que de forma exaustiva, ocasionam redução da eficácia profissional e aumento dos índices 
de absenteísmo, deixando-os predisponente para ocorrência de DORT.

A maior prevalência dos sintomas na região lombar, deve-se a importância dessa região, para o trabalho dos profissionais de enfermagem, por ser uma região de sustentação do corpo e de rotação do tronco e, muitas vezes, utilizada de forma inadequada pela má postura e por períodos prolongados (FILHO; ALMEIDA, 2016; PETERSEN; MARZIALE, 2017).

$\mathrm{Na}$ investigação realizada por YANG et al (2019), sobre a prevalência e fatores de risco de DORT na China, em 702 enfermeiros, constatou-se que a dor lombar foi o sintoma mais comumente relatado $(80,1 \%)$, seguido pela dor no pescoço $(78,6 \%)$ e no ombro $(70,4 \%)$. Estes dados corroboram com o resultado desta pesquisa, uma vez que a dor lombar é o sintoma mais citado. Em outra pesquisa realizada por SOUSA et al (2015), em 40 enfermeiros, os principais sintomas foram: lombalgia $(29,4 \%$; $\mathrm{n}=27)$, dor na cervical $(20,7 \%$; $\mathrm{n}=19)$, dor nos tornozelos e pés $(16,4 \% ; \mathrm{n}=15)$. Neste estudo a lombalgia também foi o sintoma mais relatado, entrando em consonância com os resultados encontradas na pesquisa. Num outro estudo sobre a DORT em enfermeiros, realizado em Portugal por RIBEIRO et al (2017), com uma amostra ( $n=409)$, principalmente do sexo feminino $(84,0 \%)$, observouse que a região lombar foi a região corporal mais acometida (63,1\%), seguida pela cervical, dorsal, ombros e punho/mão. Existe uma grande semelhança no que concerne a região mais afetada pelos sintomas. Outra evidência é o estudo realizado por RODRIGUES (2016), sobre os DORT em 34 profissionais de enfermagem, foi identificada a prevalência de sintomas osteomusculares entre os trabalhadores de 94,11\% nos últimos 12 meses e de $58,82 \%$ nos últimos 7 dias, com as regiões corporais mais referidas, sendo a parte inferior das costas $(79,41 \%)$, pescoço $(64,70 \%)$ e parte superior das costas $(58,82 \%)$. Neste estudo observa-se mais uma vez, uma maior prevalência dos sintomas osteomusculares na parte inferior das costas ou região lombar, o que fortalece os resultados dessa pesquisa.

A probabilidade de ter sintomas e eventualmente DORT aumenta quando os enfermeiros realizam trabalho por turnos longos, cuidados de higiene dos doentes e as ações relativas ao seu conforto na cama, sendo consideradas as tarefas com maior efeito na presença de sintomas de DORT na região lombar (SERRANHEIRA et al., 2012).

\section{CONCLUSÃO}

Após a realização do estudo concluiu-se que pouco mais da metade dos enfermeiros entrevistados apresentaram sintomas osteomusculares, sendo que na sua maioria descreveram que havia uma estreita relação destes sintomas com o exercício de enfermagem, sendo estes, casos sugestivos de distúrbios osteomusculares relacionados ao trabalho.

Os fatores desencadeantes dos distúrbios osteomusculares relacionados ao trabalho nesta pesquisa podem estar relacionados ao duplo vínculo empregatício e a carga horaria de trabalho. A região lombar foi a mais afetada por ser a região de sustentação do corpo e de rotação do tronco, sendo muitas das vezes utilizada de forma inadequada pela má postura e por períodos prolongados de trabalho. A procura de estabilidade financeira influenciou para que pouco mais da metade tivesse mais de um emprego. Durante esse processo de busca por melhores condições de vida, os enfermeiros esquecem de cuidar de seu próprio bem-estar, negligenciando e deixando-se susceptível a agravos que a longo prazo comprometem à saúde e sua qualidade de vida. Diante 
disso, é importante que os enfermeiros deste hospital compreendam e eliminem os fatores de risco que contribuem para o surgimento de dor e distúrbios, com intuito de reduzir as lesões e criar um ambiente de trabalho mais confortável.

Por isso, é necessário criar condições de trabalho adequadas, investir em programas preventivos, e organização dos serviços existentes, que garantem a melhoria da saúde dos trabalhadores, assim como o aumento da produtividade, que para além de evitar o afastamento temporário ou até mesmo definitivo dos profissionais de enfermagem, contribuirá para a redução de custos e encargos, que advém de tais complicações.

Existe uma necessidade da realização de futuros estudos em profissionais de enfermagem com uma amostra maior, em outros hospitais da região, que possibilite compreender com exatidão os fatores relacionados aos distúrbios osteomusculares no trabalho, proporcionando melhor ergonomia $\mathrm{e}$ planejamento de estratégias de trabalho que ajudem o profissional a sentir-se confortável e prestar melhores serviços.

\section{AGRADECIMENTO}

Gostaríamos de agradecer a todos enfermeiros do Hospital e pronto Socorro 28 de Agosto ManausAM, pela paciência e pela disponibilidade do seu tempo durante o processo de coleta de dados, bem como a toda equipe do hospital que direta ou indiretamente apoiou durante a realização da pesquisa, o nosso muito obrigado.

Todos os autores declararam não haver qualquer potencial conflito de interesses referente a este artigo.

\section{REFERÊNCIAS}

ALMEIDA, A. M. DE O. et al. Estresse ocupacional em enfermeiros que atuam em cuidados ao paciente crítico. Revista de Enfermagem UFPE, v. 10, n. 5, p. 1663-1671, 2016.

AMARAL, F.; TORRES, B.; CARVALHO, V. DE. Validação do Questionário Nórdico de Sintomas Osteomusculares como medida de morbidade Validity of the Nordic Musculoskeletal Questionnaire as morbidity measurement tool. Rev Saúde Pública, v. 36, n. 3, p. 307-312, 2002.

BAPTISTA, A. T. P. et al. Adoecimento de trabalhadores de enfermagem no contexto hospitalar.

Revista Enfermagem UERJ, v. 26, p. e31170, 2018.

BEZERRA, S. K. F.; COSTA, E. C. Ginástica laboral como proposta de intervenção e promoção da saúde dos profissionais de enfermagem. v. 53, n. 9, p. 1-16, 2016.

BRITO, J. C. DE P.; VENENO, J. D. C. Fatores Condicionantes Para O Adoecimento De Docentes Enfermeiros De Uma Instituição De Ensino Superior No Município De Porto Velho -Ro. Revista Saber Científico, n. 2000, p. 65-74, 2015.

CARGNIN, Z. A. et al. Non-specific low back pain and its relation to the nursing work process. Revista Latino-Americana de Enfermagem, v. 27, 2019.

COSTA, A. J. F.; DIAS, B. L. Importância de um programa de exercícios na prevenção de LER/DORT. IX Mostra de Trabalhos Acadêmicos III Jornada de Iniciação Científica. Anais...2015

CUNHA, Y. F. F.; SOUSA, R. R. GÊNERO E ENFERMAGEM: UM ENSAIO SOBRE A 
INSERÇÃO DO HOMEM NO EXERCÍCIO DA ENFERMAGEM. RAHIS, v. 13, n. 3, p. 140-149, 10 ago. 2017.

DUARTE, A. et al. Fatores de riscos para distúrbios osteomusculares relacionados ao trabalho - DORT em profissionais de enfermagem. Revista de Pesquisa: Cuidado é fundamental online, v. 2, n. 3, p. 53-56, 2012.

FELLI, V. E. A. et al. Exposição dos trabalhadores de enfermagem às cargas de trabalho $\mathrm{e}$ suas consequências. Revista da Escola de Enfermagem da USP, v. 49, n. spe2, p. 98-105, dez. 2015.

FERRI, J.; XAVIER, D. Qualidade de vida no trabalho dos profissionais de enfermagem em ambiente Hospitalar: UMA REVISÃO INTEGRATIVA. Revista epaço para saúde, v. 16, n. 1, p. 66-74, 2015.

FILHO, I. M. DE M.; ALMEIDA, R. J. DE. Estresse ocupacional no trabalho em enfermagem no Brasil: uma revisão integrativa. Revista Brasileira em Promoção da Saúde, v. 29, n. 3, p. 447-454, 2016.

FIOCRUZ/COFEN. Perfil da enfermagem no Brasil: relatório final: Brasil, 2013.

FONSECA, ROSÁRIO; SERRANHEIRA, F. Sintomatologia musculoesquelética auto-referida por enfermeiros em meio hospitalar. Revista Portuguesa de Saude Publica, v. 6, p. 37-44, 2006.

FRANÇA, M. DE F. et al. Burnout e os aspectos laborais na equipe de enfermagem de dois. Revista Latino Americana de Enfermagem, v. 20, n. 5, 2012.

LELIS, C. M. et al. Work-related musculoskeletal disorders in nursing professionals: An integrative literature review. ACTA Paulista de Enfermagem, v. 25 , n. 3, p. 477-482, 2012.

LUCCA, S. R. DE. Saúde, saúde mental, trabalho e subjetividade. Revista Laborativa, v. 1, p. 147-159, 2017.

LUCCA, S. R. DE; RODRIGUES, M. S. D. Absenteísmo dos profissionais de enfermagem de um hospital universitário do estado de São Paulo, Brasil. Revista Brasileira de Medicina do Trabalho, v. 13, n. 2, p. 76-82, 2015.

MARTINS, E. A.; CORREA, C. S. Doenças osteomusculares relacionadas ao trabalho com destaque aos profissionais da área de Enfermagem. Revista Perspectiva: Ciencia e Saúde, v. 2, n. 2, p. 107-118, 2017.

MASCARENHAS, C.; NOVAES, S. V. DE. Sintomas osteomusculares em acadêmicos dos cursos de saúde de uma universidade pública. Revista Eletrônica da Fainor, v. 8, n. 1, p. 113-131, 2015.

MATT, S. B.; FLEMING, S. E.; MAHEADY, D. C. Creating Disability Inclusive Work Environments for Our Aging Nursing Workforce. JONA: The Journal of Nursing Administration, v. 45, n. 6, p. 325-330, jun. 2015.

MELO, T. A. DE; ASSIS, M. A. DE. Riscos ocupacionais envolvendo auxiliares e técnicos de enfermagem na ESF. Revista Científica UMC, p. 14, 2018.

PAULA, E. A. DE; AMARAL, R. M. M. F. DO. Atuação interdisciplinar em grupos de qualidade de vida para pacientes com Lesões por esforços 
repetitivos/Distúrbios osteomusculares relacionados ao trabalho - LER/DORT. Revista Brasileira de Saúde Ocupacional, v. 44, 2019.

PETERSEN, R. DE S.; MARZIALE, M. H. P. Analysis of work capacity and stress among nursing professionals with musculoskeletal disorders. Revista gaucha de enfermagem, v. 38, n. 3, p. e67184, 2017.

PINHATTI, E. D. G. et al. Psychosocial aspects of work and minor psychic disorders in nursing: use of combined models. Revista Latino-Americana de Enfermagem, v. 26, p. e3068, 14 nov. 2018.

REIS, P. F. O Gênero e sua influência na prevalência de distúrbios musculoesqueléticos em Enfermeiros. Blucher Engineering Proceedings. Anais...São Paulo: Editora Blucher, dez. 2016Disponível em: $<$ http://www.proceedings.blucher.com.br/articledetails/25122>

RIBEIRO, T. et al. Work related musculoskeletal disorders in primary health care nurses. Applied Nursing Research, v. 33, p. 72-77, fev. 2017.

ROCHA, V. N. et al. Doenças ocupacionais relacionadas ao trabalho da Enfermagem. International Nursing Congress, p. 1-5, 2017.

RODRIGUES, C. MARIA DE ARAUJO. Sintomas osteomusculares relacionados ao trabalho de enfermagem em uma unidade de terapia intensiva: uma abordagem sobre ler/dort. [s.l: s.n.].

SANTOS, R. P.; ANDRADE, E. N. DE. Frequência e fatores associados dos sintomas musculoesqueléticos em auxiliares de enfermagem. Fisioter. Bras, v. 12, n. 1, p. 20-24, 2011.
SERRANHEIRA, F. et al. Lombalgias e trabalho hospitalar em enfermeiro(a)s. Revista Brasileira de Medicina do Trabalho, v. 10, n. 2, p. 80-87, 2012.

SOUSA, M. N. A. DE et al. Prevalência de distúrbios osteomusculares em enfermeiros. FIEP Bulletin online, v. 85, n. I, p. 775-780, 1 jan. 2015.

YANG, S. et al. Prevalence and Risk Factors of Work-Related Musculoskeletal Disorders Among Intensive Care Unit Nurses in China. Workplace Health and Safety, v. 67, n. 6, p. 275-287, 2019. 